\title{
BER based Performance Analysis of MCCDMA over Multipath Channels
}

\author{
Gagandeep Singh Dhaliwa \\ ECE Department \\ Lovely Professional University \\ Phagwara
}

\author{
Navpreet Kaur \\ ECE Department \\ Lovely Professional University \\ Phagwara
}

\begin{abstract}
Multi-Carrier Code Division Multiple Access (MC-CDMA) is one of the recent technologies to support multiple users with high speed data communications in advanced wireless systems. In this paper implementation and analysis of MCCDMA over different fading channel Rayleigh Fading, Rician Fading and Nakagami Fading Channel is presented. The performance is analysed and compared with DS-CDMA (Direct Sequence Code Division Multiple Access) system in terms of BER curves and spectral efficiency curves for different fading channels.
\end{abstract}

\section{Keywords}

MCCDMA, CDMA, OFDM, Rayleigh Fading, Rician Fading, Nakagami Fading.

\section{INTRODUCTION}

Multicarrier Code Division Multiple Access (MC-CDMA) is a new hybrid multiple access technology of OFDM (Orthogonal Frequency Division Multiplexing) and CDMA (Code Division Multiple Access) that has emerged to overcome the drawbacks of both OFDM and CDMA. MCCDMA provides a solution for problems for limiting current mobile systems, such as sensitivity to multipath conditions, poor bandwidth efficiency, interference and capacity issues, high signal processing complexity and inadequate performance of high-speed data transmission. MC-CDMA has emerged as another feasible option for forward-looking MC communications systems by exploiting the flexibility and potential offered by the combination of OFDM and CDMA. One large advantage of this technology is its robustness in case of multipath propagation [1]. As MC-CDMA takes the advantages of both OFDM and CDMA systems and makes an efficient transmission system by spreading the input data symbols with spreading codes in the frequency domain. It uses a number of narrowband orthogonal subcarriers with symbol duration longer than the delay spread. This makes it unlikely for all the subcarriers to be affected by the same deep fades of the channel at the same time thereby improving performance in wireless environments. Furthermore synchronization during transmission becomes easier with longer symbol durations. In this paper the BER analysis of MC-CDMA system in Rayleigh, Rician and Nakgami fading channels is presented to study its performance in these multipath channel environments and compared with DSCDMA.

Following this introduction, Section II provides a brief review of related work. The detail of system implementation is provided in Section III. Explanation of results and analysis is done in Section IV and Section V finally concludes the work done.

\section{RELATED WORK}

The literature reports various performance results of wireless communication system with MC-CDMA. [2] showed that MC-CDMA is simpler than DS-CDMA and it shows better performance than OFDM at low signal power. Some researchers have already found that the BER performance can be improved by using MC-CDMA technique rather than the DS-CDMA technique [3]. Some researchers were interested in enhancing multiplexing techniques for down link multi-user system to increase capacity, security and interference rejection capability in high data-rate transmission [4]. The literature also reports work comprising reduction of PAPR drawbacks associated with OFDM [5] by introducing RM codes. Some researchers have contributions in the analysis of MC-CDMA over different fading channels and use of different codes $[4,6]$.

In this paper we analyze the MC-CDMA capability to reduce the frequency selective fading problem in CDMA by implementing MC-CDMA in Rayleigh Fading Channel, Rician Fading Channel and Nakagami Fading channel wireless fading channels. The main contribution of this paper is to show the BER performance and spectral efficiency in MC-CDMA techniques over these fading channels (Rayleigh, Rician and Nakagami) using Walsh codes in comparison with DS-CDMA using MATLAB ${ }^{\text {TM }} 7.9$.

\section{MC-CDMA IMPLEMENTATION}

In MC-CDMA, symbols are modulated on many subcarriers to introduce frequency diversity instead of using only one carrier like in CDMA. Each user data is first spread using a given high rate spreading code in the frequency domain. A fraction of the symbol, corresponding to a chip of the spreading code, is transmitted through a different sub-carrier. The implementation is done according to the figure 1 shown below:-

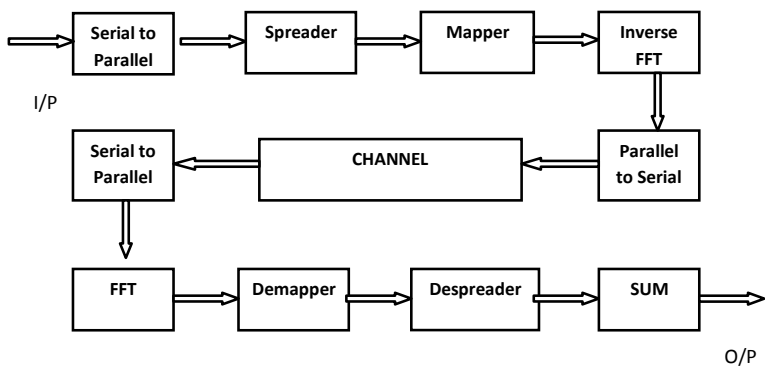

Fig 1: MC-CDMA implementation block diagram 
The serial input data stream is converted into parallel streams and are spread using CDMA spreading sequences in the frequency domain and each of them is multiplied with short spreading code to distinct between users, figure 1 . The parallel sequences are converted back to serial sequence and multiplied with long spreading code, this time to separate different cells in downlink. The spread data is mapped to $\mathrm{N}$ orthogonal subcarriers through IDFT and finally guard period is inserted to avoid ISI caused by multipath fading. Due to serial-to-parallel conversion, symbol duration at subcarrier level is $\mathrm{P}$ times longer than original symbol. This is important to retain frequency non-selective fading over each subcarrier. Otherwise, if the spreading was implemented directly on the original data sequence, the data rate could become too high and frequency selective fading would appear. The basic transmitter structure of an MC-CDMA scheme is similar to that of an OFDM scheme. The main difference is that the MCCDMA scheme transmits the same symbol in parallel through different subcarriers, whereas the OFDM scheme transmits different symbols. At the receiver side the signal is demodulated by recovering Fourier coefficients in DFT and then despread using long and short codes. The power spectrum of MC-CDMA signal is shown in figure 2.

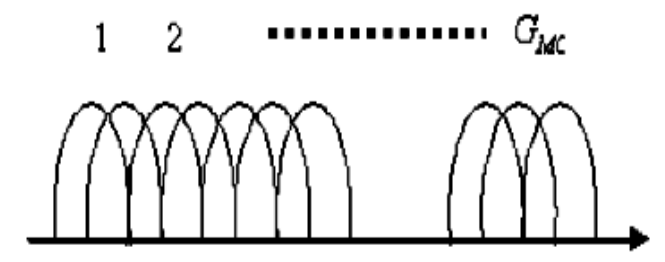

Figure 2: Power Spectrum of MC-CDMA Signal

\subsection{Fading Channels}

In wireless communication, the signal can be attenuated with time while propagating over a certain media. In wireless communication fading is mostly due to multipath propagation or shadowing which affects the wave propagation [7]. Multipath propagation occurs when a radio signal takes two or more different paths after it is transmitted from the antenna and before its reception on the receiving antenna. There are different ways of modelling a wireless communication channel that can help in modelling the important statistical properties of real world communication systems and can also give an idea of the signal amplitudes of the transmitted signals that can be expected at the receiver side. There are different fading models that can be used to estimate the fading over a channel, e.g. Nakagami fading, Log-normal shadow fading, Rayleigh fading, Rician fading, Weibull fading etc,

\subsubsection{Rayleigh Fading Channel:-}

The Rayleigh Fading Channel is mostly applied in cases when there is no LOS (Line of Sight) between the transmitter and the receiver.The channel also adds AWGN noise to the signal samples after it suffers from Rayleigh Fading. Let the received signal " $y$ " is given as:

$y=h x+n$

Where; $\mathrm{n}$ is AWGN (Additive White Gaussain Noise) with zero mean \& unit variance; $h$ is the Rayleigh Fading response with zero mean \& unit variance; $\mathrm{x}$ is the transmitted signal..The transmitted symbols $\mathrm{x}$ can be obtained from the received signal y by the process of equalization as given below:

$\hat{y}=\frac{y}{h}=\frac{h x+n}{h}=x+z$

Where $\hat{y}$ is the estimation of the received signal.

The theoretical BER for BPSK modulation scheme over Rayleigh fading channel (with AWGN noise) is given by:

$P_{\mathrm{b}}=\frac{1}{2}\left(1-\sqrt{\frac{E_{\mathrm{b}} / N_{0}}{1+E_{\mathrm{b}} / N_{0}}}\right)$

The theoretical BER for BPSK modulation scheme over an AWGN channel is given as:

$P_{b}=\frac{1}{2} \operatorname{erfc} \sqrt{\frac{E_{b}}{N_{0}}}$

\subsubsection{Rician Fading Channel:-}

The Rician Fading Channel model is used when there is a dominant Line of Sight component (LOS) between the transmitter and receiver. The LOS component is also known as Specular Component \& the multipath component is known as the Random or Scatter Component. The amplitude distribution of the specular component will have non-zero mean, whereas, the random component will have zero-mean. The Rician Factor ' $\mathrm{K}$ ' is defined as the ratio of Power of Specular component to the Power of Random component. $K=\frac{m}{2 \sigma^{2}}$

Where ' $m$ ' is mean $\&$ ' $\sigma$ ', is the variance. To simulate a Rician Fading channel, mean and sigma has to be calculated with the given Rician ' $\mathrm{K}$ ' factor. The mean \& Variance are given by:-

$m=\sqrt{K / K+1}$
$\sigma=\sqrt{\frac{1}{2 *(k+1)}}$

Theoretical BER for BPSK over Rician Fading Channel with AWGN noise is given by the following expression:

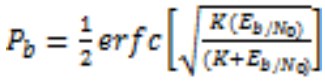

\subsubsection{Nakagami Fading Channel:-}

Nakagami distribution is capable to describe both Rayleigh and as well as Rician distributions. Unlike the Rician distribution, Nakagami distribution does not assume a LOS conditions between the transmitter and the receiver, it uses a parametric gamma distribution-based density function to describe the experimental data and get approximate distribution, the PDF of Nakagami distribution is:

$f(r)=\frac{2 m^{m} r^{2 m-1}}{\Gamma(m) \Omega^{m}} \exp \left\{\frac{-m r^{2}}{\Omega}\right\} ; m \geq \frac{1}{2}, r \geq 0$ 
Where "m" is Nakagami Fading factor, it gives the idea about fading degree during propagation of data due to the factors like scattering \& Multipath interference. When " $\mathrm{m}$ " approaches to infinity, then Nakagami Fading channel becomes a non-fading channel $\&$ " $\Omega$ " is the average power of multipath scatter field. " $\Gamma(m)$ " is the gamma function. The parameters " $\mathrm{m}$ " \& " $\Omega$ " can be calculated as:

$m=\frac{E^{2}\left[x^{2}\right]}{\operatorname{Var}[x]}$ and $\Omega=E\left[x^{2}\right]$

Basically Rayleigh \& Rician Fading channels are special cases of Nakagami fading channel model. For $m>1$, the fluctuations of the signal strength reduce compared to Rayleigh fading and with higher values of $\mathrm{m}$ less sever channels can be modeled. For $m=0$ the Nakagami acts as Rician Distribution.

\subsection{Spreading codes}

Walsh-Hadamard sequences are bipolar spreading sequences that are used for channel separation in DS-CDMA. They are easy to generate, orthogonal sequences with zero cross correlation in ideal conditions. A basic Hadamard matrix is given by $\left[\begin{array}{cc}1 & 1 \\ 1 & -1\end{array}\right]$ and an $\mathrm{N} \times \mathrm{N}$ matrix is built from this basic matrix by repetition using the formula: $\left[\begin{array}{ll}H_{N} & H_{N} \\ H_{N} & \overline{H_{N}}\end{array}\right]$

\section{Simulation Results}

The MC-CDMA system described in section III is implemented using MATLAB 7.9 [8] with various simulation parameters enlisted in Table 1 . The BER curves and spectral efficiency curves are obtained from the simulations.

Table 1: Simulation Parameters for MC-CDMA system

\begin{tabular}{|l|l|}
\hline Contents & Parameters \\
\hline Channel Types & 4 tap Rayleigh, Rician \& Nakagami-m \\
\hline No. of Users & 4 \\
\hline Modulation & BPSK $1 / 2$ \\
\hline Spreading & Walsh-Hadamard \\
\hline
\end{tabular}

Figure 3 shows the BER curve for MC-CDMA in Rayleigh, Rician and AWGN channel in comparison with CDMA for 4 users. The low BER for MC-CDMA shows the improvement over CDMA in multipath fading environments: Rayleigh channel and Rician channels.

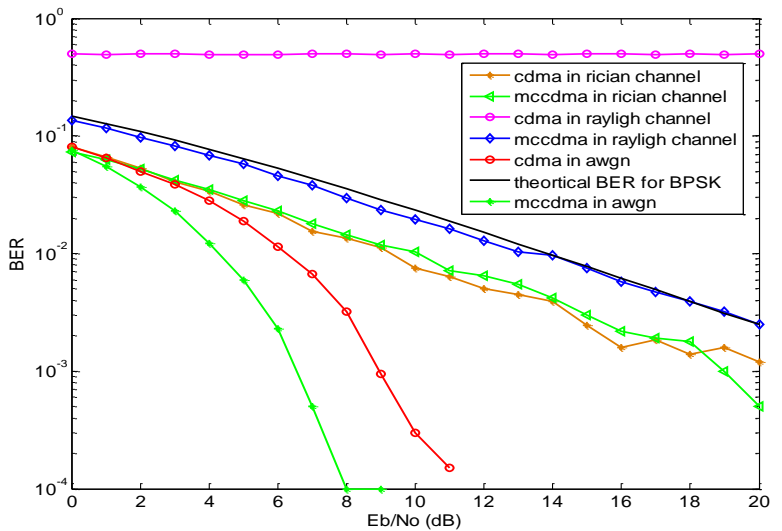

Fig 3: MC-CDMA vs CDMA in Rayleigh, Rician and AWGN channel

Figure 4 shows the performance of MC-CDMA in Nakagami channel for different values of $\mathrm{m}$. It is shown that that BER improves as the value of $\mathrm{m}$ approaches 2.5

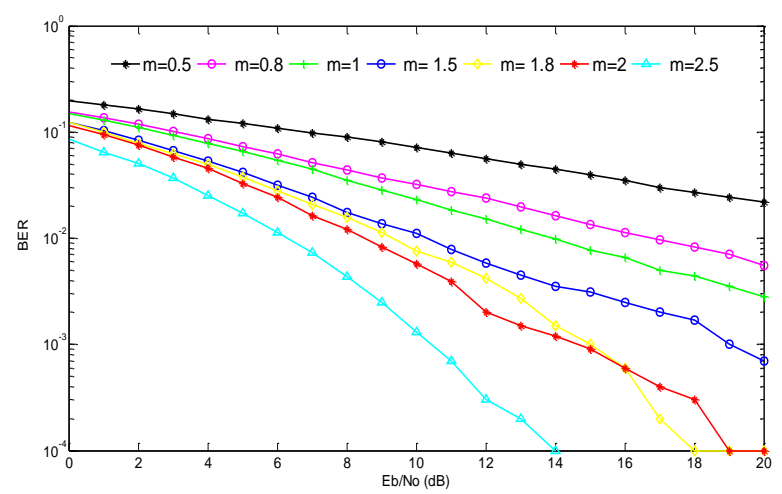

Fig 4: MC-CDMA in Nakagami channel for $m=0.5,0.8$, 1,1.5, 1.8,2 and 2.5

Figure 5 shows the performance comparison of CDMA and MC-CDMA in Nakagami channel for $m=0.8,1.5$ and 2. The BER improvement by MC-CDMA over CDMA in different channels is enlisted in Table 2 .

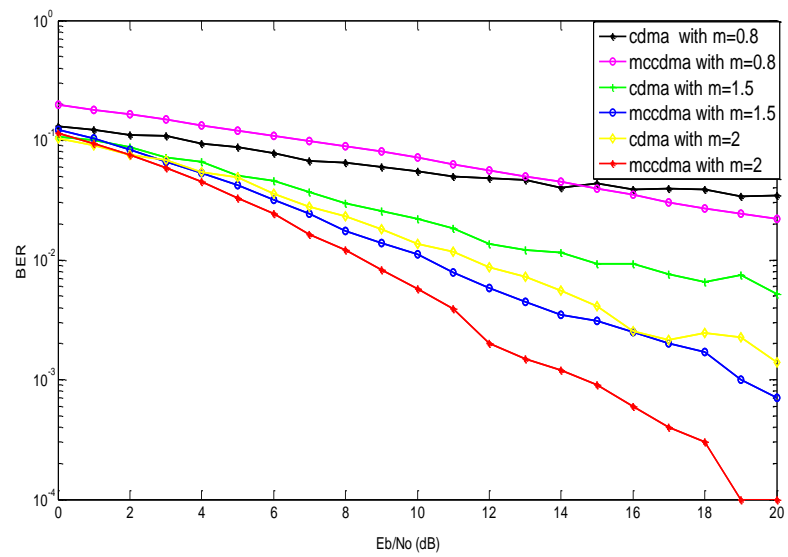

Fig 5: MC-CDMA vs CDMA in Nakagami channel $(\mathrm{m}=\mathbf{0 . 8 , 1 . 5}$ and 2$)$ 
Table 2: SNR improvement by MCCDMA over CDMA

\begin{tabular}{|l|l|l|}
\hline \multicolumn{1}{|c|}{ Channel } & BER & $\begin{array}{l}\text { SNR } \\
\text { improvement }\end{array}$ \\
\hline Rayleigh Channel & $10^{-1}$ & $20 \mathrm{~dB}$ \\
\hline Rician Channel & $10^{-1}$ & $2 \mathrm{~dB}$ \\
\hline AWGN Channel & $10^{-2}$ & $3 \mathrm{~dB}$ \\
\hline Nakagami (m=0.8) & $10^{-2}$ & $6 \mathrm{~dB}$ \\
\hline Nakagami $(\mathrm{m}=1.5)$ & $10^{-2}$ & $8 \mathrm{~dB}$ \\
\hline Nakagami $(\mathrm{m}=2)$ & $10^{-3}$ & $8 \mathrm{~dB}$ \\
\hline Nakagami $(\mathrm{m}=2.5)$ & $10^{-3}$ & $6 \mathrm{~dB}$ \\
\hline Nakagami $(\mathrm{m}=3)$ & $10^{-3}$ & $4 \mathrm{~dB}$ \\
\hline
\end{tabular}

Figure 6 shows spectral efficiency curves for MC-CDMA and CDMA in Rayleigh, Rician and Nakagami channels. It is shown that MCCDMA provides higher spectral efficiency hence higher bit rate than CDMA at the same SNR.

With the results obtained it is thus observed that MC-CDMA outperforms CDMA system both in BER and spectral efficiency for Nakagami and Rayleigh channel while for Rician channel the performance is comparable with CDMA.

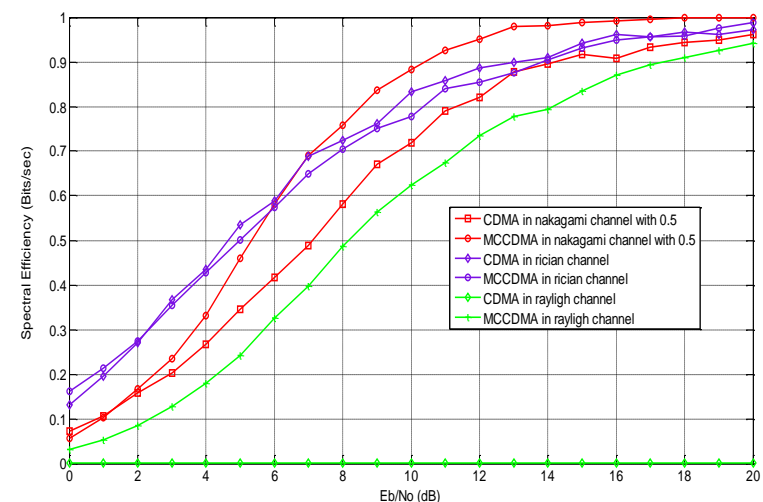

Figure 6: Spectral efficiency for MC-CDMA and CDMA in Rayleigh, Rician and Nakagami channel $(m=0.5)$.

\section{CONCLUSION}

In this paper, performance analysis of MC-CDMA is done for wireless environments by implementing MC-CDMA system model in three multipath fading channels: Rayleigh, Rician and Nakagami channels. The lower BER and higher spectral efficiency obtained for MC-CDMA system in comparison with DS-CDMA for these channels show that MC-CDMA reduces the frequency selective fading problem in CDMA; hence it is an ideal candidate for the multipath fading environments. In future, reduction of PAPR in OFDM by use of different equalisation techniques, use of different spreading codes, introduction of FEC at transmitter and receiver can be done for enhancing the performance of MC-CDMA in these environments.

\section{REFERENCES}

[1] Pragya, P. and Dutta, P. 2010, ,Muti-Carrier CDMA Overview with BPSK Modulation In Rayleigh Channel; Computer Science and Information Technology (ICCSIT), 2010 3rd IEEE International Conference on, $464-469$.

[2] Park, Y.; Kim,C.; Cho,K.; Lee,C.; Lee,H.; Kim,J.; Kwak,K. 2004, Performance of UWB DSCDMA/OFDM/MC-CDMA system.; MWSCAS '04 , Vol. 137-40.

[3] Amin, S.A. ; Alam, M. ; Majumder, S.P. 2009 Performance Analysis of Multi-carrier DS- CDMA Wireless Communication System, Computer Modeling and Simulation; Third UK Sim European Symposium.

[4] Faisal M., Haider J., Simulation Based Performance Analysis of MCCDMA and CDMA over Rayleigh Fading Channel" , International Journal on Internet and Distributed Computing Systems, vol 2, no 1, 2012,120122.

[5] Rizwan, S. 2011, Effect of using RM code to control PAPR in MCCDMA and OFDM; Multitopic Conference (INMIC), IEEE 14th International.

[6] Ghanim, M.F. , Abdullah, M.F.L. 2011, Multi-user MCCDMA using Walsh code for Rayleigh and Gaussian channel, Research and Development (SCOReD), 2011 IEEE Student Conference on, 58-63.

[7] Theodore S. Rappaport, "Wireless Communications", Prentice Hall, 2000.

[8] Communication Tool Box user guide Matlab (R2009b) 